Editorial

Urologe 2020 59 (Suppl 1):S1

https://doi.org/10.1007/s00120-020-01303-9

(C) Springer Medizin Verlag GmbH, ein Teil von Springer Nature 2020

Liebe Kolleginnen und Kollegen,

ich möchte zunächst den Mitgliedern der Programmkommission, den Vorsitzenden und Mitgliedern der Arbeitskreise und v.a. den Gutachtern und Obergutachtern für die aktive Mitgestaltung unseres 72. Kongresses der DGU danken. Wie Sie alle wissen, hat uns COVID-19 einen gewaltigen Strich durch die Rechnung gemacht, so dass wir schließlich trotz positiver Signale der Behörden in Sachsen den Präsenzkongress in Leipzig absagen mussten.

Dennoch ist es uns gelungen, mit Hilfe aller Kräfte das schon fertige Programm in eine Live-Online-Version umzuwandeln. Wir werden den Kongress als „Best of DGU 2020" von 24. bis 26. September mit anwesenden Vortragenden und Moderatoren live ins Netz übertragen. Dabei werden die Kongressteilnehmer erstmals über eine virtuelle Plattform an der Veranstaltung aktiv teilnehmen können. Diese erlaubt den Zugang zum Plenum mit allen Foren, Besuch der Industriesymposien und der virtuellen Industrieausstellung. Aber es werden auch sämtliche akzeptierte Vorträge, Videobeiträge und Studienposter abrufbar sein. Außerdem haben wir mit der Team-Akademie ein virtuelles Programm für das Pflegepersonal geschaffen.

Unsere Kongressplattform ermöglicht in den 5 anschließenden Wochen den $\mathrm{Zu}$ gang zu 10 themenbezogenen UrotubeWebinaren, wo wir jeweils am Dienstag und Donnerstag von 18.00 bis 20.30 Uhr die bisher nicht berücksichtigten Formate wie Semi-Live, Arbeitskreisforen oder auch ein berufspolitisches Forum in $\mathrm{Zu}$ -

Jens Rassweiler

Klink für Urologie und Kinderurologie SLK Kliniken Heilbronn, Universität Heidelberg, Heilbronn, Deutschland

\title{
Zum 72. Live-Online-Kongress der Deutschen Gesellschaft für Urologie e.V.
}

sammenarbeit mit dem BvDU anbieten werden. Sämtliche Beiträge sind dann noch bis zum Jahresende über die Kongressplattform abrufbar.

Besonders traurig sind wir natürlich, dass wir den zahlreichen Kollegen, die dieses Jahr einen Abstract eingereicht haben, keine bessere Präsentationsmöglichkeit bieten können. Ist es uns doch gelungen, 2020 den Trend rückläufiger Abstract-Anmeldungen mit 626 Einreichungen $\mathrm{zu}$ stoppen! Hiervon konnten wir aufgrund der hervorragenden Qualität der Beiträge 383 (61\%) annehmen. Hinzu kommen 15 Studienposter („,work in progress"). Weiterhin fällt die große Bandbreite der Abstracts auf, die den Facettenreichtum unseres Faches und unserer Gesellschaft reflektieren. Es wird auf jeden Fall wieder Vortragspreise und den Filmpreis geben. Zudem werden die Beiträge durch dieses Supplement in Der Urologe ja auch zitierfähig. Ich hoffe, dass wir dennoch diesen Schwung auch auf unsere virtuelle Plattform und natürlich auch in das Jahr 2021 nach Stuttgart mitnehmen können.

Eigentlich sollte der Kongress auch durch seine Internationalität und den persönlichen Austausch unter Kollegen herausstechen. Dies ist jetzt leider nicht möglich. Andererseits bieten sich uns allen auch neue Perspektiven. So haben wir die letzte Sitzung der Programmkommission schon als Online-Meeting durchgeführt und freuen uns jetzt schon auf die Gestaltung der virtuellen Kongressplattform und die Umsetzung unseres Hybridmodells DGU 2020 Live-online! Wir haben hier den Vorteil, dass wir neues- te Technologien der virtuellen Realität praktisch nutzen können.

Ich hoffe, dass Sie alle auch gespannt auf diese neue Kongressform sind und zahlreich daran teilnehmen werden. Abschließend gilt mein besonderer Dank dem DGU-Team in Düsseldorf und Berlin, der Firma Interplan und unserem Vorstand, ohne deren Hilfe diese Mammutprojekt nicht realisierbar wäre.

Prof. Dr. med. Dr. h.c. mult. Jens J. Rassweiler FRCS (Glasgow)

Präsident der Deutschen Gesellschaft für Urologie

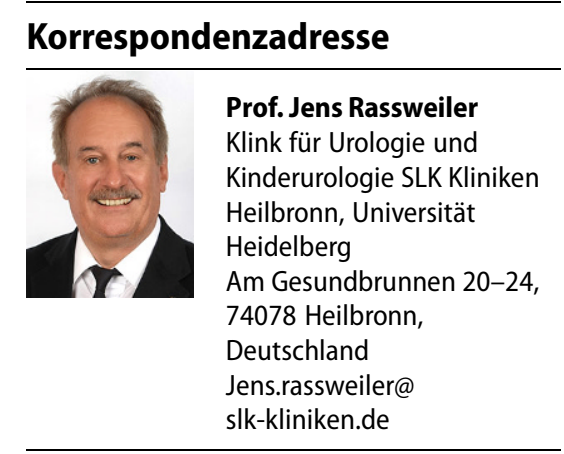

Interessenkonflikt. J. Rassweiler gibt an, dass kein Interessenkonflikt besteht. 